\title{
Clinical outcomes and complications of patients undergoing surgery during the COVID-19 incubation period
}

\author{
Mohammad Saqib Siddiqui' ${ }^{1 *}$, Bader Naji Al-Hussain², Hammad Abdulmughni Alshaikh³, \\ Mohammad Younes Alshammari ${ }^{2}$, Abdullah Mohammed Abutaleb ${ }^{4}$, \\ Amer Mohammed Alsharif ${ }^{5}$, Abdullah Abdulrahman Alwakeel ${ }^{6}$, Khaled Salem Almukhattir ${ }^{7}$,

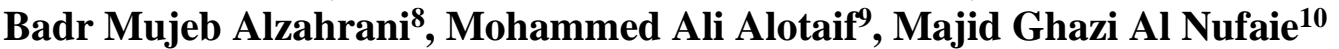

\author{
${ }^{1}$ Department of General Surgery, King Fahad General Hospital, Jeddah, Saudi Arabia \\ ${ }^{2}$ College of Medicine, Arabian Gulf University, Manama, Bahrain \\ ${ }^{3}$ Department of Emergency Medicine, King Fahad General Hosptial, Jeddah, Saudi Arabia \\ ${ }^{4}$ Department of General Surgery, Muhayil General Hospital, Muhayil, Saudi Arabia \\ ${ }^{5}$ Department of Internal Medicine, Khulais General Hospital, Khulais, Saudi Arabia \\ ${ }^{6}$ Department of Surgery, Faculty of Medicine, University of Tabuk, Tabuk, Saudi Arabia \\ ${ }^{7}$ Department of General Surgery, Ahad Rufaidah General Hospital, Abha, Saudi Arabia \\ ${ }^{8}$ College of Medicine, Medical University of Lodz, Lodz, Poland \\ ${ }^{9}$ College of Medicine, King Khalid University, Abha, Saudi Arabia \\ ${ }^{10}$ Department of Surgery, King Abdulaziz Specialist Hospital, Taif, Saudi Arabia
}

Received: 23 November 2021

Accepted: 08 December 2021

\section{*Correspondence: \\ Dr. Mohammad Saqib Siddiqui, \\ E-mail: saqib16@outlook.com}

Copyright: () the author(s), publisher and licensee Medip Academy. This is an open-access article distributed under the terms of the Creative Commons Attribution Non-Commercial License, which permits unrestricted non-commercial use, distribution, and reproduction in any medium, provided the original work is properly cited.

\begin{abstract}
Conducting surgery might be challenging during the pandemic, especially for COVID-19 patients. This is because of the high transmissibility rate of the infection, which might lead to spreading the infection. Moreover, surgery might be associated with further risk over affected patients, a significant contributor to the operated patients' impaired immunity and generalized inflammatory state. Surgery is usually associated with an increased risk of high-stress levels and a generalized inflammatory state flare-up. The present literature review discusses the mortality rates and associated factors for COVID-19 patients during the incubation period. Our findings show that these patients have higher mortality rates as surgery influences the release of high levels of proinflammatory cytokines. Besides, surgery might impair the immune functions leading to progressive deterioration of COVID-19. On the other hand, it has been reported that COVID-19 can also worsen the outcomes and increase the risk of morbidities and mortality among patients undergoing surgery. Different factors have been identified to contribute to this risk, including old age, being male, and the presence of comorbidities. Therefore, avoiding surgery during infection is suggested to reduce the risk of mortality, especially among patients with multiple risk factors. However, it should be noted that this evidence needs further validation.
\end{abstract}

Keywords: SARS-CoV-2, Death, Mortality, Complications, COVID-19, Surgery

\section{INTRODUCTION}

Since the COVID-19 pandemic was announced, thousands of reports have been published to evaluate the different factors associated with severe disease complications and mortality. ${ }^{1,2}$ These reports aimed to enhance the burdens over affected individuals by helping to innovate different interventional approaches. The disease has been reported following severe acute respiratory syndrome coronavirus 2 (SARS-CoV-2). The 
virus has been reported to cause different pathological changes to the bodies of the affected patients, leading to a variety of clinical presentations and complications. Different pulmonary and extra-pulmonary complications were evidenced in the literature. . $^{3,4}$

The presence of complications has been associated with a significant deterioration in affected patients' health, which might end up with death. In addition, conducting surgery might be challenging during the pandemic, especially for COVID-19 patients. This is because of the high transmissibility rate of the infection, which might lead to spreading the infection. Moreover, surgery might be associated with further risk over affected patients, a significant contributor to the operated patients' impaired immunity and generalized inflammatory state. ${ }^{5-7}$ In the present study, we aim to conduct a literature review to discuss the complications of patients undergoing surgery during the COVID-19 incubation period based on findings from the previous studies in the literature.

\section{LITERATURE REVIEW}

This literature review is based on an extensive literature search in Medline, Cochrane, and EMBASE databases which was performed on $27^{\text {th }}$ November 2021 using the medical subject headings (MeSH) or a combination of all possible related terms, according to the database. To avoid missing potential studies, a further manual search for papers was done through Google Scholar while the reference lists of the initially included papers. Papers discussing the emerging SARS-CoV-2 variants in the Middle East were screened for useful information. No limitations were posed on date, language, age of participants, or publication type.

\section{DISCUSSION}

\section{Overview}

Despite the major advances in the management of COVID-19 after vaccines have been introduced, no specific treatment modalities have been proposed. The current treatment guidelines only include conservative approaches aiming to reduce the severity of the affected patients. Accordingly, disease severity following infection is greatly dependant on the immune status of the affected patients. In this context, COVID-19 has been more severe, with more reported complications among individuals with impaired immune functions, including older patients and patients with underlying chronic diseases. ${ }^{8}$ Accordingly, these patients are at a higher risk of death from COVID-19-related complications than others. The impact of surgery on the generalized body health condition has been well-described. Evidence shows that surgical procedures influence the development of a significant inflammatory state and remarkably impair the immune functions of the affected patients. ${ }^{9,10}$ Infections affecting the lungs (similar to what is associated with SARS-CoV-2 and the Middle East respiratory syndrome
Coronavirus or MERS-CoV infections) also contribute to the deterioration in the affected patients' health status. Reports show that these infections influence proinflammatory chemokines and cytokines and increase neutrophil and macrophage infiltration levels. ${ }^{11-13}$ In this context, well-established evidence indicates that severe COVID-19 conditions and mortality are associated with the presence of high levels of proinflammatory cytokines. ${ }^{14}$

\section{Outcomes and complications}

COVID-19 infection can induce significant stress to the affected patients and is associated with severe morbidities and increased mortality rates. Therefore, different studies have investigated the outcomes among patients undergoing surgeries during COVID-19. Besides, studies reported the impact of surgery on the consequences of asymptomatic patients when the surgery has been performed. Therefore, this might give an insight into the role of surgery on the outcomes and prognosis of COVID-19. Among the relevant studies, Lei et al included 34 patients that had evidence of exposure to SARS-CoV-2 infection being indicated to have the surgery. ${ }^{15}$ It has been reported that none of these patients developed any symptoms before the surgical procedure. However, following the surgery, patients began to report symptoms, and COVID-19 was diagnosed in all of them by the polymerase chain reaction technique. The authors reported that $20.6 \%$ of the included patients died secondary to COVID-19-related complications. This rate has been estimated to be higher than the previously estimated all-cause mortality rates for COVID-19 patients without surgeries (2.3\%). Moreover, evidence shows that these rates are higher than the all-cause mortality rate for non-COVID-19 patients undergoing noncardiac surgical procedures $(7.9 \%) .^{16,17}$ It should be noted that the inclusion of patients in this investigation was based on the history of exposure to SARS-CoV-2, and no confirmatory tests were conducted.

Among dead patients in these settings, it has been estimated that the average duration of death after the first symptom was reported postoperatively at 8.7 days (ranging between 4 and 13 days). ${ }^{15}$ However, other reports also said shorter durations, indicating the wide variation among reports. ${ }^{14,18}$ This can be attributed to the severity of symptoms among COVID-19 patients and the presence of comorbidities which might worsen the prognosis and increase the risk of mortality among highrisk patients. In a previous investigation by Huang et al the median duration between hospital admission and intensive care unit (ICU) admission and the onset of symptoms was 7 and 10.5 days, respectively. ${ }^{14}$ The authors furtherly reported that $15 \%$ of the included participants in this study died. Although the authors did not report the mean duration between death and onset of symptoms, it is expected to be more than 10.5 days (i.e., after admission to the ICU). Accordingly, it can be suggested that patients undergoing surgical procedures 
might be prone to more stress. This can significantly progress COVID-19 and exacerbate the associated manifestations, leading to severe COVID-19 and mortality.

Different investigations also reported the outcomes of COVID-19 patients undergoing surgical procedures. For example, a previous retrospective investigation by Nahshon et al reported that $27.5 \%$ of COVID-19 patients died postoperatively. ${ }^{19}$ This has been attributed to primarily pulmonary complications secondary to COVID19. Moreover, the authors also showed that the infection occurred perioperatively. Thus, the harmful events observed in these settings are mutual between the effect of COVID-19 and surgical procedures. As we previously reported that surgery significantly impacts the outcomes of COVID-19, evidence also shows that COVID-19 can also alter the outcomes following surgical procedures. Increased risk of peri-operative complications and mortality has been demonstrated among both symptomatic and asymptomatic COVID-19 patients undergoing surgeries.

This has been indicated in a previous case-control study conducted in Italy. The authors reported that the rate of 30-day mortality from COVID-19 was remarkably higher than the estimated rate of 30-day mortality for nonCOVID-19 patients. The authors furtherly demonstrated that the risk of other postoperative complications was also significant for COVID-19 patients, especially thrombotic complications. $^{20}$ In the same context, a previous systematic review also aimed to formulate evidence regarding a combined mortality rate from the different related studies in the literature. Abate et al estimated that the mortality rate for COVID-19 patients following surgical operations was $20 \% .^{21}$ Besides, they showed that the rate of postoperative admission to the ICU was $15 \%$. Another similar meta-analysis was also conducted to investigate similar outcomes. Wang et al analyzed the findings of 47 studies in the literature, with 269 patients recruited in these studies. ${ }^{22}$ It has been reported that $49 \%$ of the included population were women, and the mean age for COVID-19 patients at the time of surgery was 50.91 years old. The authors reported that among the included 269 COVID-19 patients, only 28 died perioperatively and owing to COVID-19-related complications. Therefore, the estimated all-cause mortality rate in this study was $6 \%$ only. It has been furtherly reported that the majority of the non-survivors had progressive acute respiratory distress syndrome and/or respiratory failure. Evidence from various investigations also supports these findings..$^{8,22-25}$

The impact of surgery on the outcomes of infected patients presenting in the incubation period was also investigated in a previous investigation during the MERS$\mathrm{CoV}$ pandemic. Seddiq et al reported a case of a 61-yearold man that was indicated to have elective cardiac bypass surgery. ${ }^{26}$ The authors reported that the patient did not have a positive MERS-CoV infection on admission and did not have any laboratory abnormalities despite being diagnosed with diabetes and hypertension. However, the authors reported that MERS-CoV-related symptoms were observed during the $2^{\text {nd }}$ day after surgery. Besides, the patient tested positive for MERS-CoV infection six days after the surgery and died within the $9^{\text {th }}$ day. This might be attributed to the fact that conducting the surgery might have exacerbated the infection in this patient.

\section{Factors predicting mortality and other complications}

Different parameters were previously investigated as potential factors contributing to the development of COVID-19 severe disease and mortality. Elevated Creactive protein levels and lymphopenia were reported to be the most typical abnormalities related to death among patients undergoing surgical procedures during the incubation period of COVID-19. In the same context, higher levels of neutrophils and white blood cells are associated with an increased risk of admission to the ICU in these patients. In addition to estimating the mortality rates following surgery for COVID-19 patients, Wang et al also analyzed the most typical peri-operative surgical complications in these patients. ${ }^{22}$ The authors estimated that the most common postoperative complications included developing at least one sign of acute kidney damage, cardiopulmonary failure, myalgia or weakness, fatigue, fever, dyspnea, shortness of breath, and respiratory failure. Extreme lymphopenia was also reported among some patients. The authors estimated that the presence of either of these factors was significantly associated with the increased risk of mortality among their included population $(\mathrm{r}=0.891, \mathrm{p}<0.001)$. A less severe impact over the mortality risk was reported among other variables, including myalgia, fatigue, cough, and fever $(r=0.675, p=0.023)$. It has been further evidenced that the rates of postoperative deaths and respiratory complications among COVID-19 patients urge healthcare professionals to take adequate precautions to avoid getting infected from these patients. The rates of postoperative complications were also notable among healthcare professionals. Evidence shows the high rates of infections among these individuals. However, it was also reported that having adequate safety measures and level-3 biosafety protection is significantly associated with a reduced risk of catching infections among this personnel. ${ }^{22,27-29}$

The risk of death and presurgical complications was also correlated with various factors in a previous study. Doglietto et al reported that SARS-CoV-2 infection was positive in $19.5 \%$ of patients five days after the surgery and $80.5 \%$ of the included patients before the surgery. ${ }^{20}$ In addition, the authors demonstrated that COVID-19 increased the risk of mortality. This has been concluded as the mortality rates for COVID-19 patients were significantly higher among patients without COVID-19 [odds ratio $(\mathrm{OR})=9.5 ; 95 \%$ confidence interval $(\mathrm{CI})=1.77$ 96.53]. Increased risk of developing complications and 
death was also significantly correlated with age. A 1.04 higher rate of OR for developing complications was noticed considerably with an increase in age by one year. Being male and $\geq 70$ years of age is significantly correlated with developing COVID-19 related complications and mortality $(\mathrm{OR}=1.75$, and 2.30, respectively; $\mathrm{p}<0.0001)$. Other reported factors that can predict mortality and peri-operative complications included major over minor surgeries, malignant over benign diagnosis, American Society of Anesthesiologists grades 3-5 over 1-2, and emergency over elective surgeries. ${ }^{30}$ Moreover, evidence shows that having a history of medical conditions (including diabetes, hypertension, cardiac, and pulmonary diseases) might also contribute to severe disease and mortality.

The risk of mortality was also reported to be significantly high following thrombotic and pulmonary complications. Therefore, surgical procedures, especially the major ones, should be minimized during the COVID-19 pandemic, especially for the most vulnerable population, as a primary approach to reduce the secondary burdens and complications. ${ }^{30}$ Moreover, there is no doubt that the risk of mortality is higher among infected patients than others. Accordingly, it has been suggested that patients with confirmed/suspected COVID-19 diagnosis should delay their surgical plans until they have recovered from COVID-19. Only severe and urgent cases should be managed with surgical approaches.

\section{CONCLUSION}

Surgery is usually associated with an increased risk of high-stress levels and a generalized inflammatory state flare-up. Patients have higher mortality rates as surgery influences the release of high levels of proinflammatory cytokines. Besides, surgery might impair the immune functions leading to progressive deterioration of COVID19. On the other hand, it has been reported that COVID19 can also worsen the outcomes and increase the risk of morbidities and mortality among patients undergoing surgery. Different factors have been identified to contribute to this risk, including old age, being male, and the presence of comorbidities. Therefore, avoiding surgery during infection is suggested to reduce the risk of mortality, especially among patients with multiple risk factors. However, it should be noted that this evidence needs further validation.

\section{Funding: No funding sources \\ Conflict of interest: None declared \\ Ethical approval: Not required}

\section{REFERENCES}

1. Bulut C, Kato Y. Epidemiology of COVID-19. Turk j med sci. 2020;50(SI-1):563-70.

2. Zhai P, Ding Y, Wu X, Long J, Zhong Y, Li Y. The epidemiology, diagnosis and treatment of COVID19. Int j antimicrobial agents. 2020;55(5):105955.
3. Huang $\mathrm{X}$, Wei $\mathrm{F}, \mathrm{Hu} \mathrm{L}$, Wen $\mathrm{L}$, Chen $\mathrm{K}$. Epidemiology and clinical characteristics of COVID19. Arch Iranian med. 2020;23(4):268-71.

4. Gupta A, Madhavan MV, Sehgal K. Extrapulmonary manifestations of COVID-19. Nature Med. 2020;26(7):1017-32.

5. Balch CM, Shanafelt T. Combating stress and burnout in surgical practice: a review. Advances in surgery. 2010;44:29-47.

6. Helander EM, Webb MP, Menard B. Metabolic and the Surgical Stress Response Considerations to Improve Postoperative Recovery. Current pain headache rep. 2019;23(5):33.

7. Desborough JP. The stress response to trauma and surgery. British journal of anaesthesia. 2000;85(1):109-117.

8. Chen N, Zhou M, Dong X. Epidemiological and clinical characteristics of 99 cases of 2019 novel coronavirus pneumonia in Wuhan, China: a descriptive study. Lancet (London, England). 2020;395(10223):507-13.

9. Amodeo G, Bugada D, Franchi S. Immune function after major surgical interventions: the effect of postoperative pain treatment. $\mathbf{J}$ pain res. 2018;11:1297-305.

10. Ni Choileain N, Redmond HP. Cell response to surgery. Arch surg (Chicago Ill: 1960). 2006;141(11):1132-40.

11. Zhou J, Chu H, Li C. Active replication of Middle East respiratory syndrome coronavirus and aberrant induction of inflammatory cytokines and chemokines in human macrophages: implications for pathogenesis. J infect dis. 2014;209(9):1331-42.

12. Law HK, Cheung CY, Ng HY. Chemokine upregulation in SARS-coronavirus-infected, monocytederived human dendritic cells. Blood. 2005;106(7):2366-74.

13. Chien JY, Hsueh PR, Cheng WC, Yu CJ, Yang PC. Temporal changes in cytokine/chemokine profiles and pulmonary involvement in severe acute respiratory syndrome. Respirology (Carlton, Vic). 2006;11(6):715-22.

14. Huang C, Wang Y, Li X. Clinical features of patients infected with 2019 novel coronavirus in Wuhan, China. Lancet (London, England). 2020;395(10223):497-506.

15. Lei S, Jiang F, Su W. Clinical characteristics and outcomes of patients undergoing surgeries during the incubation period of COVID-19 infection. E-Clin Med. 2020;21:100331.

16. Wu Z, McGoogan JM. Characteristics of and Important Lessons From the Coronavirus Disease 2019 (COVID-19) Outbreak in China: Summary of a Report of 72314 Cases From the Chinese Center for Disease Control and Prevention. JAMA. 2020;323(13):1239-42.

17. Kumar P, Renuka MK, Kalaiselvan MS, Arunkumar AS. Outcome of Noncardiac Surgical Patients Admitted to a Multidisciplinary Intensive Care Unit. Indian j cri care med. 2017;21(1):17-22. 
18. Wang D, Hu B, Hu C. Clinical Characteristics of 138 Hospitalized Patients With 2019 Novel CoronavirusInfected Pneumonia in Wuhan, China. JAMA. 2020;323(11):1061-9.

19. Nahshon C, Bitterman A, Haddad R, Hazzan D, Lavie O. Hazardous Postoperative Outcomes of Unexpected COVID-19 Infected Patients: A Call for Global Consideration of Sampling all Asymptomatic Patients Before Surgical Treatment. World j surg. 2020;44(8):2477-81.

20. Doglietto F, Vezzoli M, Gheza F. Factors Associated With Surgical Mortality and Complications Among Patients With and Without Coronavirus Disease 2019 (COVID-19) in Italy. JAMA surg. 2020;155(8):691702.

21. Abate SM, Mantefardo B, Basu B. Postoperative mortality among surgical patients with COVID-19: a systematic review and meta-analysis. Patient safety surg. 2020;14:37.

22. Wang $\mathrm{K}, \mathrm{Wu} \mathrm{C}, \mathrm{Xu} \mathrm{J}$, et al. Factors affecting the mortality of patients with COVID-19 undergoing surgery and the safety of medical staff: A systematic review and meta-analysis. EClinicalMedicine. 2020;29:100612.

23. Seeliger B, Philouze G, Cherkaoui Z, Felli E, Mutter D, Pessaux P. Acute abdomen in patients with SARS-CoV-2 infection or co-infection. Langenbeck's arch surg. 2020;405(6):861-6.

24. Son PT, Reda A, Viet DC. Exchange transfusion in the management of critical pertussis in young infants: a case series. Vox sanguinis. 2021;116(9):976-82.

25. Nguyen TM, Huan VT, Reda A. Clinical features and outcomes of neonatal dengue at the Children's
Hospital 1, Ho Chi Minh, Vietnam. J clin virol. 2021;138:104758.

26. Seddiq N, Al-Qahtani M, Al-Tawfiq JA, Bukamal N. First Confirmed Case of Middle East Respiratory Syndrome Coronavirus Infection in the Kingdom of Bahrain: In a Saudi Gentleman after Cardiac Bypass Surgery. Case rep infect dis. 2017;2017:1262838.

27. El-Qushayri AE, Dahy A, Reda A. A closer look to the high burden of the psychiatric disorders among health care workers (HCWs) in Egypt during COVID-19 outbreak: A meta-analysis of 3137 HCWs. Epidemiol health. 2021:e2021045.

28. El-Qushayri AE, Ghozy S, Reda A, Kamel AMA, Abbas AS, Dmytriw AA. The impact of Parkinson's disease on manifestations and outcomes of COVID19 patients: A systematic review and meta-analysis. Reviews med virol. 2021:e2278.

29. Thieu H, Bach Dat B, Nam NH. Antibiotic resistance of Helicobacter pylori infection in a children's hospital in Vietnam: prevalence and associated factors. Minerva medica. 2020;111(5):498-501.

30. COVIDSurg Collaborative. Mortality and pulmonary complications in patients undergoing surgery with perioperative SARS-CoV-2 infection: an international cohort study. Lancet (London, England). 2020;396(10243):27-38.

Cite this article as: Siddiqui MS, Al-Hussain BN, Alshaikh HA, Alshammari MY, Abutaleb AM, Alsharif AM et al. Clinical outcomes and complications of patients undergoing surgery during the COVID-19 incubation period. Int J Community Med Public Health 2022;9:497-501. 